\title{
Desarrollo de competencias para la dirección, gestión y administración pública desde el aprendizaje servicio
}

\section{Portillo-Poblador, Nuria ${ }^{a}$; Martín-Marín, Jorge ${ }^{b}$}

${ }^{a}$ Dpto. de Estadística e Investigación Operativa Aplicadas y Calidad. Universitat Politècnica de València, nportillo@eio.upv.es, b ${ }^{b}$ pto. de Estadística e Investigación Operativa Aplicadas y Calidad. Universitat Politècnica de València,jmartinm@eio.upv.es.

\begin{abstract}
The CTALENT education innovation and improvement team has been working since 2015 on education for sustainable development from the university classrooms under the prism of the UN 2030 Agenda and, in collaboration with other organizations, to promote the development of competences in the students of the Univesrsitat Politècnica de València.

Often, we work with the Learning-Service methodology, in a concise way it could be said that the students contribute improvements to society, contribute to the common good, while they learn specific and transversal competences of their subjects together with their teaching staff. This methodology favors the learning of competencies for public management and allows students to become aware of the effects that their decisions will have on citizens from positions of management, management and public administration.
\end{abstract}

The last experiences developed in the degree of Management and Public Administration are "Ludopathy in numbers" and "The 2030 Agenda from transparency and open data" in the subjects of Introduction to statistics and Statistics applied to public administration and "Girls and children auditors ISO 9001 in Red Cross "in the subject of Quality Management.

Keywords: service learning, public management, statistics, quality management, 2030 Agenda, sustainable development objectives, specific competences, transversal competences, education for sustainable development, $N G D O$, common good

\section{Resumen}

El equipo de innovación y mejora educativa CTALENT viene trabajando desde 2015 en la educación para el desarrollo sostenible desde las aulas universitarias bajo el prisma de la Agenda 2030 de Naciones Unidas y, en colaboración con otras organizaciones para fomentar el desarrollo de competencias en los y las estudiantes de la Univesrsitat Politècnica de València.

A menudo, se trabaja con la metodología Aprendizaje-Servicio, de forma concisa podría decirse que los y las estudiantes aportan mejoras a la sociedad, contribuyen al bien común, mientras aprenden competencias especificas y transversales de sus asignaturas junto a su profesorado. Esta 
metodología favorece el aprendizaje de competencias para la dirección y gestión pública y permite al alumnado tomar conciencia de los efectos que tendrán sus decisiones en la ciudadanía desde puestos de dirección, gestión y administración pública.

Las últimas experiencias desarrolladas en el grado de Gestión y Administración Pública son "La ludopatía en cifras" y "La Agenda 2030 desde la transparencia y los datos abiertos" en las asignaturas de Introducción a la estadística y Estadística aplicada a la administración pública y "Niñas y niños auditores ISO 9001 en Cruz Roja” en la asignatura de Gestión de calidad.

Palabras clave: aprendizaje servicio, gestión pública, dirección pública, estadística, gestión de calidad, Agenda 2030, objetivos de desarrollo sostenible, competencias específicas, competencias transversales, educación para el desarrollo sostenible, ONGD, bien común. 


\section{Contexto global}

El grado de Gestión y Administración Pública (GGAP) de la Facultad de Administración y Dirección de Empresas (FADE) de la Universitat Politècnica de València (UPV) forma a sus alumnos y alumnas en competencias que les permitan desarrollar su futuro profesional en puestos de trabajo vinculados a la gestión, administración y dirección pública (Alcover, 2015) y también perfiles profesionales dirigidos al sector privado (Romano, 2016).

Tal y como se presenta en Portillo-Poblador (2017), en un mundo globalizado, es necesario poner en valor la Educación para el desarrollo sostenible (EpD) (UNECE, 2015), la Educación para la Ciudadanía Global (CONGDE, 2013) y la Agenda 2030 (NACIONES UNIDAS, 2015). En esta última se presenta una visión del mundo para el año 2030 de la que derivan 17 Objetivos de Desarrollo Sostenible (ODS) con sus 169 metas e indicadores, unos medios de implementación y un marco para el seguimiento. El logro de estos ODS depende, en gran medida, de las Políticas Públicas Educativas que los países lleven a cabo y como estas repercutan en la educación de las actuales y futuras generaciones.

La Universidad busca la formación integral de los y las estudiantes que abarque el aprendizaje de las competencias específicas propias del título y también de competencias transversales que les serán útiles en su vida profesional, personal y de ciudadanía. Esto se ve reflejado en el Plan Estratégico 2015-2020 (UPV, 2015a) y en el Proyecto Institucional de Competencias Transversales (CT) de la Universitat Politècnica de València (UPV, 2015b). Se trabajan trece competencias transversales, tabla 1, comunes a todas las titulaciones. Recientemente se ha hecho una modificación de la definición de la competencia "Conocimiento de problemas contemporáneos" incluyendo de manera explícita los ODS de la Agenda 2030. El aprendizaje de competencias pone el foco en el alumnado, centrando el proceso en lo que aprenden, así se redefine el proceso de enseñanza-aprendizaje como un proceso de aprendizaje-enseñanza.

Es importante tener en cuenta que el conjunto de competencias específicas, generales del GGAP junto con las competencias transversales, cubren las competencias del modelo propuesto por el Comité de Esquema de Certificación de Directivos Públicos Profesionales categorías 2 y 3 (2015). En Portillo-Poblador (2018) se estudia la relación entre las comtetencias del GGAP y las Las Habilidades Esenciales de Alta Dirección (HEAD).

Tabla 1. Competencias Transversales UPV

\begin{tabular}{ll}
\hline \multicolumn{1}{c}{ Código } & \multicolumn{1}{c}{ Tipo } \\
\hline CT-01 & Comprensión e integración \\
CT-02 & Aplicación y pensamiento práctico \\
CT-03 & Análisis y resolución de problemas \\
CT-04 & Innovación, creatividad y emprendimiento \\
CT-05 & Diseño y proyecto \\
CT-06 & Trabajo en equipo y liderazgo \\
\multicolumn{2}{c}{ Esta obra está bajo una Licencia Creative Commons CC BY-NC-ND 4.0 } \\
\multicolumn{2}{c}{ EDITORIAL UNIVERSITAT POLITĖCNICA DE VALÈNCIA }
\end{tabular}




\begin{tabular}{ll} 
CT-07 & Responsabilidad ética, medioambiental y profesional \\
CT-08 & Comunicación efectiva \\
CT-09 & Pensamiento crítico \\
CT-10 & Conocimiento de problemas contemporáneos \\
CT-11 & Aprendizaje permanente \\
CT-12 & Planificación y gestión del tiempo \\
CT-13 & Instrumental específica \\
\hline
\end{tabular}

En este entorno glocal, resulta imprescindible definir estrategias de aprendizajeenseñanaza orientadas al desarrollo sostenible en las titulaciones universitarias, no existen dos planetas Tierra. Si se pretende conseguir resultados diferentes, habrá que actuar de forma diferente. Una de las metodologías que pueden tener un papel relevante es el Aprendizaje-Servicio (ApS) (Aramburuzabala, 2014) de especial interés para los y las profesionales con vocación de servicio público, orientados a ser una parte importante en la resolución de los grandes retos que se presentan en la sociedad actual y de futuro.

\section{Objetivos y experiencias}

El objetivo de estas experiencias es que los alumnos y las alumnas desarrollen competencias transversales y específicas de manera integrada y orientadas al desarrollo sostenible.

Se relatan en este trabajo tres de las últimas experiencias que se han realizado con la metodología ApS:

(i) La ludopatía en cifras. La experiencia lleva dos años funcionando en las asignaturas de Introducción a la estadística (IE) y Estadística aplicada a la administración pública (EAAP) en el primer y el segundo cuatrimestre respectivamente, de segundo curso del GGAP. Se utiliza la metodología de Aprendizaje-Servicio bajo la perspectiva de incidencia o denuncia social de un problema contemporáneo que afecta a nuestra sociedad a todos los niveles y edades.

(ii) La Agenda 2030 desde la transparencia y los datos abiertos. Se ha trabajdo en este curso en las asignaturas de IE y EAAP en segundo del GGAP. Se utiliza la metodología de Aprendizaje-Servicio bajo la prespectiva de incidencia o denuncia social y pretende dar protagonismo a la Agenda 2030 desde el paradigma de Gobierno Abierno.

(iii) Niñas y niños auditores ISO 9001 en Cruz Roja. La experiencia se ha desarrollado en la asignatura de Gestión de calidad (GC) de tercer curso del GGAP de segundo cuatrimestre. Se utiliza la metodología de AprendizajeServicio bajo la perspectiva de servicio indirecto. Se realiza en colaboración con la ONGD Cruz Roja. 


\section{Metodología}

La metodología educativa utilizada en esta experiencia ha sido el aprendizaje-servicio (ApS). Con esta metodología mientras el alumno desarrolla competencias específicas y transversales vinculadas a las asignaturas, realiza un servicio a la sociedad, que puede entenderse como una contribución al bien común.

El aprendizaje-servicio, la educación para el desarrollo sostenible y las competencias transversales, constituyen una vía hacia sociedades sostenibles (Portillo-Poblador, 2017) y por este motivo resulta una metodología muy adecuada si se pretende formar de manera integrada, con actividades auténticas, profesionales, personas y ciudadanía en cualquier campo profesional pero especialmente en los perfiles orientados al servicio público y participes desde las AAPP a tener un papel fundamental en la resolución de los problemas y retos que afectan a la sociedad a nivel local y global.

Para aplicar la metodología de ApS es necesario que el alumnado aprenda contenidos propios de la aisgnaturas a la vez que ofrece un servicio a la sociedad. Existen varios tipos de servicios:

- Servicio directo: El alumnado trabaja directamente con las personas que van a recibir el servicio

- Servicio indirecto: El alumnado trabaja con materiales o documentación que después utilizarán las personas o colectivos destinatarios del servicio

- Incidencia social: El alumnado y/o profesorado detectan un problema en la sociedad y pretenden visibilizarlo, que se tome conciencia del mismo

- Servicio investigación: El alumnado trabaja e investiga sobre un problema con la finalidad de que un colectivo utilice su investigación para resolver, conocer o aportar luz sobre dicho problema

En las tres experiencias que se presenta se realiza aprendizaje de competencias específicas vinculadas a cada una de las asignaturas. En el caso del proyecto (i), las competencias específicas son las propias de la estadística centradas en los conceptos de probabilidad y esperanza matemática, en el caso del proyecto (ii) son las derivadas de la recogida de datos en forma de variables para su análisis posterior, mientras que en el proyecto (iii), las competencias específicas son las de gestión de calidad y se centran en las auditorías de calidad según la norma ISO 9001.

En cuanto a las competencias transversales, la metodología ApS permite su aprendizaje y tal y como se estudia en Portillo-Poblador (2017), existe una elevada correspondencia entre las competencias transverales y las competencias que se aprenden con la metodología ApS (Rubio, 2009).

En las experiencias que se presentan son varias las CT que se trabajan, pero únicamente se evalúan aquellas en las que las asignaturas son punto de Control, en concreto: Aplicación y pensamiento práctico (CT-02), Responsabilidad ética, medioambiental y profesional (CT-07), Pensamiento crítico (CT-09) y Conocimiento de problemas 
contemporáneos (CT-10). Otras competencias transversales que también se trabajan son: Comprensión e integración (CT-01), Diseño y proyecto (CT-05), Trabajo en equipo y liderazgo (CT-06), Comunicación efectiva (CT-08) y Aprendizaje permanente (CT-11).

En la tabla 2, se muestran para cada una de las experiencias, el aprendizaje, el tipo de servicio, el servicio y en la tabla 3 se presentan las fases de las experiencias:

Tabla 2. Experiencias: Aprendizaje, tipo de servicio y servicio

\begin{tabular}{|c|c|c|c|}
\hline & La ludopatía en cifras & $\begin{array}{l}\text { La Agenda } 2030 \\
\text { desde la } \\
\text { transparencia y los } \\
\text { datos abiertos }\end{array}$ & $\begin{array}{l}\text { Niñas y niños } \\
\text { auditores ISO } 9001 \text { en } \\
\text { Cruz Roja }\end{array}$ \\
\hline $\begin{array}{l}\text { Aprendiza } \\
\text { je }\end{array}$ & $\begin{array}{l}\text { Contextualizar los } \\
\text { conceptos de } \\
\text { probabilidad y } \\
\text { esperanza matemática } \\
\text { vinculados a los juegos } \\
\text { de azar }\end{array}$ & $\begin{array}{l}\text { Definir variables e } \\
\text { indicadores con la } \\
\text { visión de la Agenda } \\
2030 \text { desde los datos } \\
\text { abiertos }\end{array}$ & $\begin{array}{l}\text { Profundizar en los } \\
\text { procedimientos de } \\
\text { calidad ISO } 9001 \text { y en } \\
\text { la preparación de } \\
\text { documentación para } \\
\text { auditoría interna }\end{array}$ \\
\hline $\begin{array}{l}\text { Tipo de } \\
\text { Servicio } \\
\end{array}$ & Incidencia social & Incidencia social & Servicio indirecto \\
\hline Servicio & $\begin{array}{l}\text { Visibilizar y } \\
\text { concienciar el } \\
\text { problema de la } \\
\text { ludopatía a los jóvenes }\end{array}$ & $\begin{array}{l}\text { Visibilizar el potencial } \\
\text { de los datos abiertos } \\
\text { para contribuir a la } \\
\text { Agenda } 2030\end{array}$ & $\begin{array}{l}\text { Auditoria interna } \\
\text { realizada por niños y } \\
\text { niñas con los } \\
\text { materiales elaborados } \\
\text { por el alumnado del } \\
\text { GGAP para que los } \\
\text { niños y niñas tomen } \\
\text { conciencia de su } \\
\text { entorno y como } \\
\text { respetarlo y cuidarlo }\end{array}$ \\
\hline
\end{tabular}

El proyecto (i) pretende informar/concienciar a los jóvenes de la actual problemática de la ludopatía agravada por la enorme influencia de las apuestas en internet o en los salones de apuestas a pie de calle, así como en la publicidad engañosa y las estrellas que aparecen en los anuncios para promocionar las apuestas o las consecuencias legales que genera en los y las jóvenes suplantar la personalidad de otros adultos para acceder a estas apuestas. El alumnado trabaja los contenidos de probabilidad y esperanza matemática desde los juegos de azar. La ludopatía es considerada una enfermedad sobre la que es necesario poner en alerta a los y las jóvenes y también a las personas adultas.

El proyecto (ii) pretende dar a conocer las implicaciones de la normativa de transparencia en ayuntamientos, así como la situación de los datos abiertos en las AAPP. El alumnado revisa el cumplimiento de la normativa de transparencia y lo evalúan y proponen modificaciones sobre algunos conjuntos de datos abiertos o nuevos conjuntos de datos abiertos que a disposición de la ciudadanía a la vez que contribuyan al seguimiento de la Agenda 2030. En las variables a recoger, se definen con detalle, en el caso de variables 
cualitativas se definen las distintas clase de las variables, la periodicidad con la que se actualizarían, etc., en definitiva plantéan como recoger información para después realizar análisis estadísticos que permitan obtener información relevante para tomar decisiones abaladas por los datos, con especial interés para el seguimiento de las metas de los ODS de la Agenda 2030.

El proyecto (iii) pretende contribuir a la educación para el desarrollo sostenible de las nuevas generaciones, en concreto, contribuye a concienciar a los niños y niñas del trabajo que los profesionales del servicio de prevención de playas realizan día a día para asegurar la conservación de las playas y garantizar la seguridad de las personas incluso las que tienen dificultades de movilidad. Serán los niños y niñas quienes realicen la auditoria interna según la norma de gestión de calidad ISO 9001 para seis procedimientos clave del servicio de prevención de playas de CR. Este proyecto se acompaña con formación específica en auditorías internas para el alumnado de GGAP por parte de responsables de CR y con formación para las niñas y niños auditores sobre los sistemas de gestión de calidad y las auditorias a cargo de responsables de CR y del profesorado de la asignatura de GC. Finalmente las niñas y niños auditores realizan la auditoría interna en dos playas de la Comunidad Valenciana.

Tabla 3. Experiencias: Fases

\begin{tabular}{|c|c|c|c|}
\hline Fases & La ludopatía en cifras & $\begin{array}{l}\text { La Agenda } 2030 \text { desde } \\
\text { la transparencia y los } \\
\text { datos abiertos }\end{array}$ & $\begin{array}{l}\text { Niñas y } \quad \text { niños } \\
\text { auditores ISO } 9001 \text { en } \\
\text { Cruz Roja }\end{array}$ \\
\hline $\begin{array}{l}\text { Buscar } \\
\text { organizació } \\
\text { n externa } \\
\text { para } \\
\text { colaborar o } \\
\text { público al } \\
\text { que } \\
\text { difundir } \\
\end{array}$ & $\begin{array}{l}\text { CEP e IES } \\
\text { principalmente }\end{array}$ & $\begin{array}{l}\text { CEP e IES } \\
\text { principalmente }\end{array}$ & Cruz Roja \\
\hline $\begin{array}{l}\text { Acordar } \\
\text { proyecto } \\
\text { común para } \\
\text { desarrollar }\end{array}$ & $\begin{array}{l}\text { La ludopatía como } \\
\text { problema } \\
\text { contemporáneo en la } \\
\text { sociedad, } \\
\text { principalmente en } \\
\text { jóvenes }\end{array}$ & $\begin{array}{l}\text { La importancia de los } \\
\text { datos abiertos y la } \\
\text { transparencia para la } \\
\text { Agenda } 2030\end{array}$ & $\begin{array}{l}\text { Documentación para } \\
\text { auditorias internas de } \\
\text { calidad del servicio } \\
\text { prevención de playas } \\
\text { de Cruz Roja }\end{array}$ \\
\hline
\end{tabular}




\begin{tabular}{|c|c|c|c|}
\hline $\begin{array}{l}\text { Desarrollar } \\
\text { los } \\
\text { contenidos } \\
\text { y } \\
\text { materiales }\end{array}$ & $\begin{array}{l}\text { Investigación amplia: } \\
\text { probabilidad de ganar } \\
\text { en un juego de azar; } \\
\text { consecuencias legales } \\
\text { de suplantar la } \\
\text { personalidad de un } \\
\text { adulto, implicaciones } \\
\text { de los gobiernos, } \\
\text { publicidad engañosa, } \\
\text { el efecto de las } \\
\text { estrellas deportivas en } \\
\text { los anuncios de } \\
\text { apuestas, etc. }\end{array}$ & $\begin{array}{l}\text { Revisión de los datos } \\
\text { abiertos publicados por } \\
\text { las administraciones y } \\
\text { del cumplimiento de la } \\
\text { normativa de } \\
\text { transparencia. } \\
\text { Modificar conjuntos de } \\
\text { datos abiertos ya } \\
\text { publicados para que } \\
\text { contribuyan a la } \\
\text { Agenda } 2030 \text { y diseñar } \\
\text { nuevos conjuntos de } \\
\text { datos }\end{array}$ & $\begin{array}{l}\text { Elaboración de la } \\
\text { documentación para } \\
\text { auditoria interna ISO } \\
\text { 9001de seis } \\
\text { procedimientos clave } \\
\text { de gestión de calidad } \\
\text { adaptada a niños y } \\
\text { niñas entre } 9 \text { y } 12 \text { años }\end{array}$ \\
\hline $\begin{array}{l}\text { Realizar el } \\
\text { Servicio }\end{array}$ & $\begin{array}{l}\text { Exposición de los } \\
\text { alumnos y las alumnas } \\
\text { del GGAP en los CEP } \\
\text { e IES y otros } \\
\text { colectivos para } \\
\text { concienciar a otros y } \\
\text { otras jóvenes sobre el } \\
\text { problema de la } \\
\text { ludopatía }\end{array}$ & $\begin{array}{l}\text { Exposición de los } \\
\text { alumnos y las alumnas } \\
\text { del GGAP en los CEP } \\
\text { e IES y otros para } \\
\text { concienciar a otras } \\
\text { personas sobre como } \\
\text { contribuir a la Agenda } \\
2030 \text { desde las AAPP }\end{array}$ & $\begin{array}{l}\text { Auditoria interna en } \\
\text { dos playas de la } \\
\text { comunidad Valenciana } \\
\text { realizada por niños y } \\
\text { niñas con los } \\
\text { materiales elaborados } \\
\text { por el alumnado del } \\
\text { GGAP para que los } \\
\text { niños y niñas tomen } \\
\text { conciencia de su } \\
\text { entorno, como } \\
\text { respetarlo y cuidarlo }\end{array}$ \\
\hline
\end{tabular}

\section{Resultados}

Las experiencias se trabajan de manera obligatoria por todo el alumnado de manera que los y las estudiantes conozcan el potencial de esta metolodogía que permite ayudar a otros directa o indirectamente.

En cuanto a los particpantes en los proyectos:

(i) En dos años han participado en el proyecto 28 equipos de 3-4 estudiantes, dos profesores y alumnado de otros centros. En la Figura 1 se muestra el extracto de alguno de los materiales elaborados por el alumnado y la difusión del proyecto en un IES de València en uno de los momentos de mayor atención en el que se explica las consecuencias legales de suplantar la personalidad de un adulto.

(ii) En un año han participado 23 equipos de estudiantes, dos profesores y personas y alumnado externo con los que han compartido el trabajo. En la Figura 2 se muestra el cartel de difusión de la experiencia en la Casa del pueblo e UGT y una imagen de la difusión momentos antes de comenzar.

(iii) En el proyecto han participado 6 equipos de estudiantes, dos profesores, varios responsables de CR, 10 niños y niñas, dos voluntarios de CR que acompañaban en todo momento a los niños y niñas y el personal de CR en cada una de las playas. En la Figura 3 se muestra un extracto de los materiales generados por el alumnado y algunas imágenes de la formación específica sobre auditorías Esta obra está bajo una Licencia Creative Commons CC BY-NC-ND 4.0 
internas recibida en locales de CR Valencia y un momento de la auditoria interna realizada por nñas y niños auditores junto al personal de CR en la playa de Oliva.

En la Figura 4 se muestra uno de los carteles de difusión para los proyectos (i) y (ii).
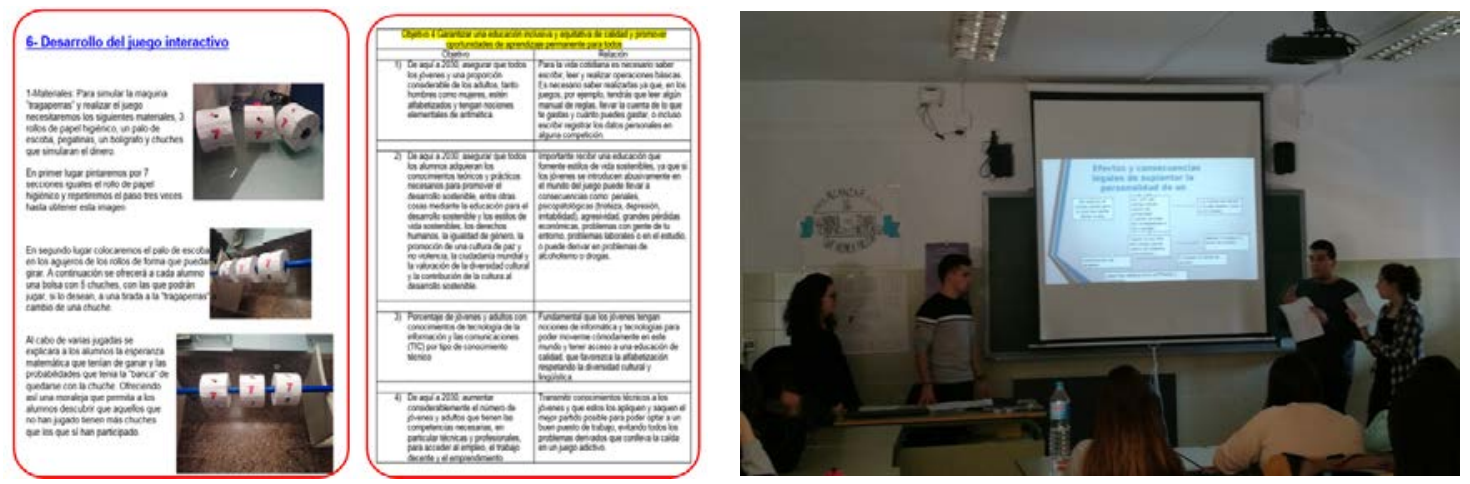

Fig. 1 Proyecto La ludopatía en cifras. (1a) Extracto de la presentación de distintos proyectos. (1b) Difusión del proyecto en un IES de València, en pantalla, efectos legales de suplantar la personalidad de un adulto.
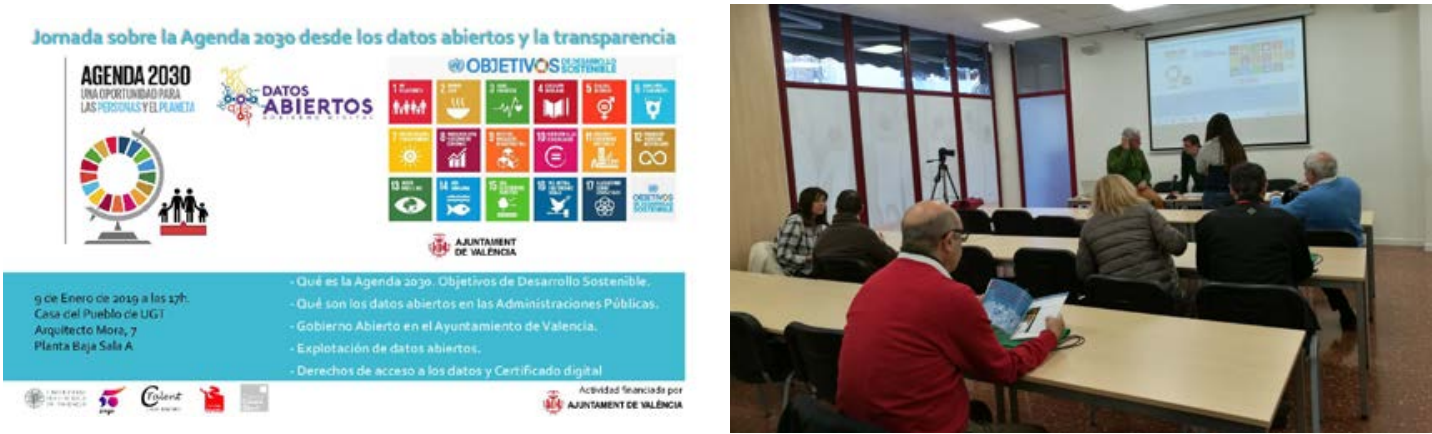

Fig. 2 Proyecto La Agenda 2030 desde los datos abiertos y la transparencia. Difusión de la experiencia en Casa del Pueblo de UGT. (2a) Cartel de difusión, (2b) Preparando difusión del proyecto

En cuanto al aprendizaje del alumnado:

(i) Han contextualizado y puesto en práctica los conceptos de probabilidad y esperanza matemática vinculados a los juegos de azar. Adicionalmente, al trabajar en la ludopatía desde otros aspectos, han aprendido que es una enfermedad, los problemas que genera en el entorno familiar, social, de trabajo o estudio, el papel de las AAPP en sus políticas, la publicidad engañosa vinculada a las apuestas, la influencia de las grandes estrellas, principalmente del mundo del deporte, en los anuncios de apuestas. Han relacionado el proyecto con los ODS de la Agenda 2030, en ocasiones llegando al detalle de las metas de los ODS, lo que les ha permitido aprender sobre desarrollo sostenible. Algo que les será de gran utilidad en su futuro puesto de trabajo.

(ii) El alumnado ha trabajo en el diseño de conjuntos de datos abiertos definiendo las variables a registrar, el tipo de variable, las clases para las variables cualitativas, el tiempo de actualización de los datos. También han definido indicadores con la visión de la Agenda 2030. Han revisado si las AAPP 
estudiadas cumplían la normativa de transparencia y la relación de cada obligación con la Agenda 2030. También han aprendido sobre desarrollo sostenible al igual que el alumnado del proyecto (i).

(iii) Han adaptado seis procedimientos de procesos clave según la norma de getión de calidad ISO 9001 para que resulten sencillos de entender a las niñas y los niños auditores. Han profundizado en las auditorías internas y en la documentación necesaria para pasar la auditoría. Contribuyen a incluir la educación para el desarrollo sostenible de las niñas y niños en el ámbito de la educación no formal.
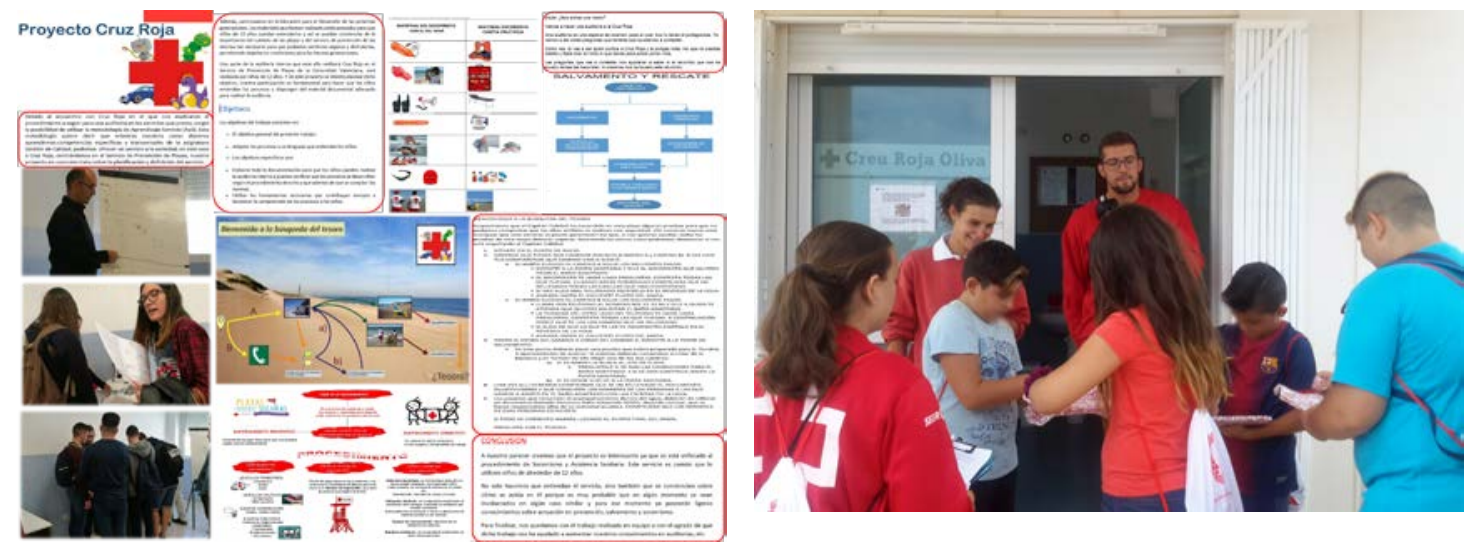

Fig. 3 Proyecto Niñas y niños auditores ISO 9001 en Cruz Roja. (3a) Extractos de los materiales para la auditoria del proyecto y formación específica sobre auditorías internas en locales de CR Valencia. (3b) Momento de la auditotoría interna en la Playa de Oliva.

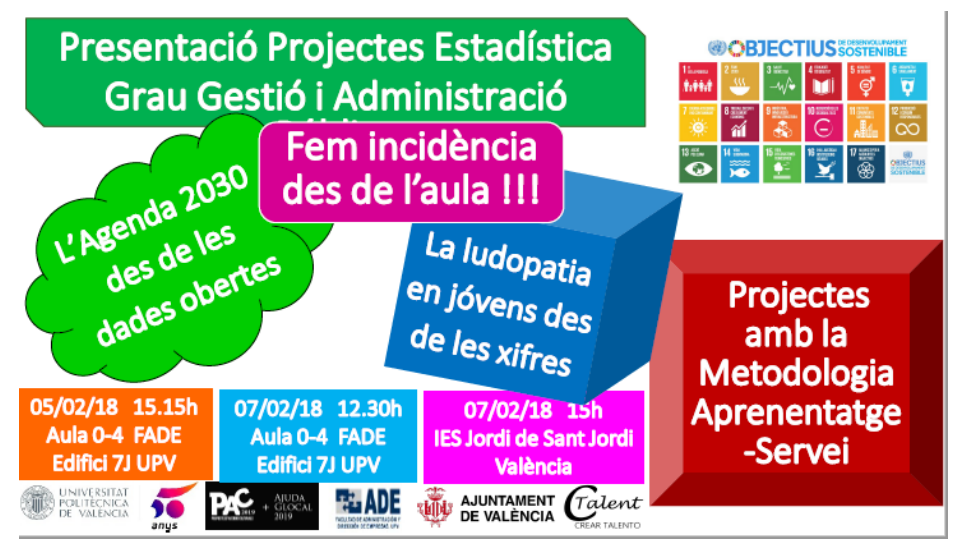

Fig. 4 Cartel de la difusión de los proyectos la Ludopatía en cifras y La Agenda 2030 desde los datos abiertos y la transparencia

Uno de los mejores resultados que se obtiene al utilizar el ApS como metodología de aprendizaje-enseñanza es justo el servicio que implica la metodología y como produce un cambio de $360^{\circ}$ en como se produce el aprendizaje. El alumnado además de aprender y pensar en superar las asignaturas, deja de pensar en si mismo para pensar en quien recibirá el servicio, cual será la mejora que producirá en la sociedad, el reto al que podrán dar 
solución o arrojar luz para esclarecer situaciones de conflicto. Esta parte es decisiva para cualquier estudiante de grado o máster, pero es especialmente significativo para el alumnado de GGAP por las implicaciones sociales que conlleva su perfil profesional de servicio público, orientado a la ciudadanía, centrado en resolver los grandes retos a las que se enfrenta la humanidad, muchos de los cuales se concentran en la Agenda 2030.

Los resultados en cuanto al servicio de los proyectos son:

(i) La experiencia ayuda a visibilizar y concienciar el problema de la ludopatía a los jóvenes desde otros jóvenes. Las voces cercanas, de estudiantes a estudiantes, con corta diferencia de edad, causa un efecto que no sería posible esperar si la difusión la realizara una persona adulta, independientemente del colectivo a la que perteneciera.

(ii) La experiencia ayuda a visibilizar el potencial de los datos para tomar decisiones informadas a partir de su análisis y pone en valor los datos abiertos que producen las AAPP y que pueden reutilizarse con distintos fines por la ciudadanía o por otros colectivos. En concreto, en esta experiencia se orienta su uso para diseñar indicadores que contribuyan a la Agenda 2030.

(iii) La experiencia contribuye a que las niñas y niños auditores conozcan el papel que realizan los profesionales del servicio de playas en su día a día. Cuidan de las playas y de la seguridad de las personas que hacen uso de ellas. Además, se ocupan de que sean accesibles a las personas con movilidad reducida. Los niños y niñas aprendieron que es una auditoría interna, cual es su importancia. Indicar que en la auditoría interna detectaron una no conformidad justo en el procedimiento de baño asistido, el que permite que personas con movilidad reducida puedan también disfrutar del mar, lo cual contribuyo a que las niñas y niños tomarán conciencia sobre la situación de las personas con movilidad reducida. Podría decirse que el alumnado de GGAP ha conseguido incluir la educación para el desarrollo sostenible en el ámbito de educación informal fomentado que los niños y niñas tomen conciencia de su entorno, de como respetarlo y cuidarlo

\section{Conclusiones}

Al valorar los resultados de las experiencias y los aprendizajes realizados, se han encontrado dificultades y aspectos positivos. Sin lugar a dudas, los aspectos positivos inclinan la balanza significativamente.

En relación con las dificultades encontradas, podemos indicar que giran en torno a la gestión del tiempo:

- Gestionar adecuadamente los tiempos en la universidad y en las organizaciones es complicado. Sin duda, un punto crítico de los proyectos. Realizar actividades 
vinculadas a las asignaturas y hacer coincidir los tiempos de las asignaturas con los tiempos de organizaciones externas es laborioso y no siempre fructífero

- El profesorado dedica significativamente más tiempo al diseño y coordinación de este tipo de actividades que, a otras tareas, no menos importantes vinculadas al desarrollo de las mismas, no obstante, el resultado del aprendizaje y del servicio es prácticamente siempre positivo para todas las partes involucradas

Los aspectos positivos más relevantes del uso de la metodología de Aprendizaje-Servicio son:

- El ApS fortalece el proceso de Aprendizaje-Enseñanza del alumnado bajo el paradigma del desarrollo de competencias específicas y transversales mediante actividades auténticas que forman parte de organizaciones reales

- El ApS fomenta una universidad permeable en la que tanto alumnado como profesorado traspasan los muros de las aulas y laboratorios y aprenden en armonía con la sociedad que les rodea

- Contribuye al aprendizaje profundo de los estudiantes al cambiar la manera de cómo enfrentan su aprendizaje

- El proyecto (ii) está vinculado directamente con las AAPP, en concreto ayuntamientos, por tanto el beneficio es doble, no afectando exclusivamente a las AAPP, sino también a la ciudadanía

- Uno de los apartados a desarrollar en los distintos proyectos es evaluar la relación entre cada proyecto y la Agenda 2030. Esto ha permitido que todo el alumnado participante conozca la Agenda 2030 con sos ODS, metas e indicadores. Con ello se pretende que los perfiles futuros en puesto de dirección, gesión y administración pública hayan trabajado ya con la Agenda 2030 y sean por tanto, conscientes de su carácter integrador en las AAPP

\section{Agradecimientos}

Durante el tiempo de ejecución de los proyectos, el equipo ha obtenido financiación para difusión o para realizar los servicios a través de los proyectos:

- Desarrollo de actividades alineadas con la Agenda 2030 para el aprendizaje de competencias desde las asignaturas, y en colaboración con diferentes organizaciones, financiado por el Vicerrectorado de Estudios, Calidad y Acreditación (VECA) de la Universitat Politècnica de València (UPV) en la convocatoria Proyectos de Innovación y Mejora Educativa (PIME) en la convocatoria 2017-2018 en dos años

- Los Objetivos de Desarrollo Sostenible en asignaturas bajo el prisma Glocal financiado por el Centro de Cooperación al Desarrollo (CCD) de la UPV en la convocatoria Glocal 2018 
- Escritores y escritoras responsables. Carta a la UNESCO de la UPV bajo el prisma de los ODS financiado por el Área de Cultura de la UPV de la convocatoria Propuestas de Acciones Culturales (PAC) en la convocatoria 2018

- La Agenda 2030 en las aulas universitarias como instrumento para el aprendizaje de competencias para el desarrollo sostenible financiado por el Centro de Cooperación al Desarrollo (CCD) de la UPV en la convocatoria Glocal 2019

- Científicos y científicas responsables en la UPV con la Agenda 2030 financiado por el Área de Cultura de la UPV de la convocatoria Propuestas de Acciones Culturales (PAC) en la convocatoria 2019

- La Agenda 2030 desde los Datos Abiertos y la Transparencia en un proyecto de Apredizaje-Servicio en la Universitat Politècnica de València en la convocatoria de ayudas a proyectos de educación y fomento de la cultura de la transparencia y el gobierno abierto en la convocatoria de 2018

- Ayudas de FADE para la asistencia a congresos en las convocatorias 2017 y 2018

\section{Referencias}

ARAMBURUZABALA, P. (2014). Aprendizaje-servicio. Ciudadanía activa, justicia social y aprendizaje. En V. Ballesteros Alarcón (Coord.). Implicaciones de la educación y el voluntariado en la formación de una ciudadanía activa. Perspectiva internacional (pp 33-48). Granada: GEU.

COMITÉ DE LOS ESQUEMAS DE CERTIFICACIÓN DE DPP CATEGORÍAS 2 Y 3 (2015). El Sistema de Certificación de Directivos Públicos Profesionales (SC/DPP). Esquemas de Categorías 2 y 3. 2. Directia Occasional Paper. Nº 2. Serie: dirección pública profesional, técnico-científica.

CONGDE. (2013). Educación para el desarrollo. Una estrategia de cooperación Imprescindible. <http:/guiarecursos-epd.coordinadoraongd.org/uploads/documentos/ que_es_la_educacion_para_el_desarrollo.pdf $>$. [Consulta: 8 de febrero de 2017].

DEELEY, S. J. (2016). El Aprendizaje-Servicio en educación superior: Teoría, práctica y perspectiva crítica (Vol. 44). Narcea Ediciones.

HANNING, A., ABELSSN, A.P., LUNDQVIST, U., SVANSTR€OM, M., (2012). Are we educating engineers for sustainability? Comparison between obtained competences and Swedish industry's needs. Int. J. Sustain. High. Educ. 13 (3)) (pp 305-320).

INTERED. (2014). Educación para el desarrollo y la ciudadanía global (EpDCG). Guía para su integración en centros educativos. <http://redciudadaniaglobal.org/wpcontent/uploads/2011/11/publicacion-EpDCG_vFinal.pdf $>$. [Consulta: 8 de febrero de 2017]. 
GERMAIN, F.; PÉREZ-RICO, C. (2014). La educación por competencias como medio para facilitar la toma de control del aprendizaje por el estudiante. FEM: Revista de la Fundación Educación Médica, 2014, 17 (1), p. 11-19.

LÓPEZ-PASTOR, V.M. (2011) El papel de la evaluación formativa en la evaluación por competencias: aportaciones de la red de evaluación formativa y compartida en docencia universitaria. Revista de Docencia Universitaria, 9 (1), Enero-Abril 2011, 159-173.

MAYOR ZARAGOZA, F. (2015). "Universidades y ODS" en E-DHC, Quaderns Electrònics sobre el Desenvolupament Humà i la Cooperació, 5, p. 8-11.

MURGA-MENOYO, M.A. (2015). Competencias para el desarrollo sostenible: las capacidades, actitudes y valores meta de la educación en el marco de la Agenda global post-2015. Foro de Educación, 13 (19), p. 55-83.

NACIONES UNIDAS. (2015). Transformar nuestro mundo: la Agenda 2030 para el Desarrollo Sostenible, 2015. <http://unctad.org/meetings/es/SessionalDocuments/ ares70d1_es.pdf $>$. [Consulta: 8 de febrero de 2017].

NACIONES UNIDAS (2017). Objetivos de Desarrollo Sostenible (ODS). $<$ http://www.un.org/sustainabledevelopment/es/action2015/ $>$. [Consulta: 1 de junio de 2017].

OXFAM INTERMÓN, UNICEF. (2015). España frente a los retos de la agenda de desarrollo sostenible. $<$ https://oxfamintermon.s3.amazonaws.com/sites/default/ files/documentos/files/EspanaRetosDesarrolloSostenible.pdf>. [Consulta: 8 de febrero de 2017].

PAUSCH, M. (2016). Citizenship education in times of crisis. Foro de Educación, 20, p. 3-9.

PORTILLO-POBLADOR, N. (2017). El aprendizaje-servicio, la educación para el desarrollo sostenible y las competencias transversales, una vía hacia sociedades sostenibles. VIII Congreso Nacional y III Internacional De Aprendizaje-Servicio Universitario. APS-U8. SEVILLA2017.

PORTILLO-POBLADOR, N. ; MARTIN-MARIN ; J., ALONSO CAMINERO, J.M. (2017). Educación para el desarrollo sostenible de las futuras generaciones desde las aulas universitarias. Una experiencia en colaboración con Cruz Roja. Conferencia internacional RED-U 2017. Bilbao. 13-14 de noviembre 2017.

PORTILLO-POBLADOR, N. (2018). Acreditación de competencias en el grado de Gestión y Administración Pública para los futuros directivos públicos. La gestión del talento en las organizaciones. Ed. Wolters Club p. 223-231.

RED ESPAÑOLA DEL PACTO MUNDIAL DE NACIONES UNIDAS. (2016). El sector privado ante los ODS. Guía práctica para la acción. Madrid, $<$ http://www.pactomundial.org/wp- 
content/uploads/2017/02/Guia_ODS_PM_20170215_web.pdf $>$. [Consulta: 8 de febrero de 2017].

ROMANO ÁLVAREZ, C. (2016). Adecuación de las capacidades incluidas en el grado de gestión y administración pública al correcto desempeño profesional en el sector privado. Trabajo Final de Grado. Valencia: Universitat Politècnica de València, < https://riunet.upv.es/handle/10251/68542 > [Consulta: 7 de mayo 2019].

RUBIO, L. (2009). El aprendizaje en el aprendizaje servicio. En J. M. Puig (Coord.), R. Batlle, C. Bosch, M. de la Cerda, T. Climent, M. Gijón... J. Trilla, Aprendizaje servicio (ApS): Educación y compromiso cívico (pp. 91-105). Barcelona: Graò.

UE4SD. (2013). Empowering educators for a sustainable future Strategy for Education for Sustainable Development Tools for policy and practice workshops on competences in education for sustainable development. <http:/www.unece.org/fileadmin/ DAM/env/esd/Images/Empowering_Educators_for_a_Sustainable_Future.pdf $>$.

[Consulta: 17 de octubre de 2016].

UNECE. (2012) Learning for the future: Competences in Education for Sustainable Development. $\quad<$ http://www.unece.org/fileadmin/DAM/env/esd/ESD_Publications/ Competences_Publication.pdf $>$ [Consulta: 5 de abril de 2016].

UNECE. (2015). Ten years of the UNECE strategy for education for sustainable development. Evaluation report on the implementation of the UNECE Strategy for Education for Sustainable Development from 2005 to 2015. United Nations. ECE/CEP/179. $\quad<$ http://www.unece.org/fileadmin/DAM/env/esd/11thMeetSC/ Documents/ECE.CEP.179_-_10_years_of_Learning.pdf $>$ [Consulta: 10 de junio de 2016].

UNESCO (2014). Declaración de Aichi-Nagoya sobre la Educación para del Desarrollo Sostenible. Conferencia Mundial Aichi-Nagoya (Japón), 10-12 de noviembre.

UPV. (2015a). Plan Estratégico 2015-2020. <https://www.upv.es/noticiasupv/documentos/plan_estrategico_upv2020.pdf>. [Consulta: 22 de junio de 2017].

UPV. (2015b). Proyecto Institucional Competencias Transversales. $<$ http://www.upv.es/contenidos/COMPTRAN/ >. [Consulta: 22 de junio de 2017].

VAZQUEZ TOLEDO, S; REVILLA CARRASCO, A; LIESA ORUS, M (2015). El Aprendizaje-Servicio: diálogo Universidad y Sociedad (No. BOOK-2015-003). $<$ https://zaguan.unizar.es/record/31885/files/El\%20Aprendizaje-

Servicio\%20:\%20di\%C3\%A1logo\%20Universidad\%20y\%20Sociedad.pdf>. [Consulta: 10 de junio de 2016]. 